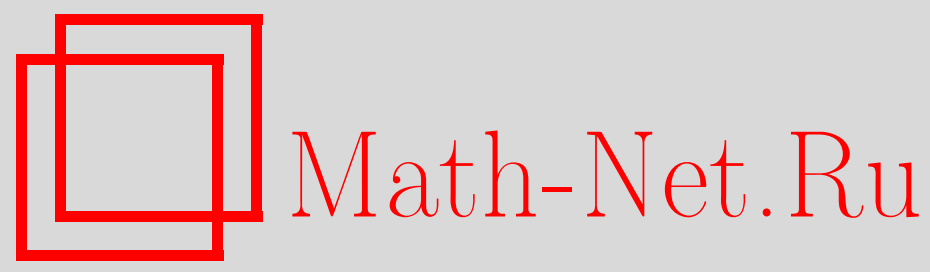

Г. Е. Иванов, Седловая точка для дифференциальных игр с сильно выпукло-вогнутым интегрантом, Матем. заметки, 1997, том 62, выпуск 5, 725-743

DOI: https://doi.org/10.4213/mzm1659

Использование Общероссийского математического портала Math-Net.Ru подразумевает, что вы прочитали и согласны с пользовательским соглашением http://www.mathnet.ru/rus/agreement

Параметры загрузки:

IP : 3.85 .73 .92

26 апреля 2023 г., $15: 27: 51$

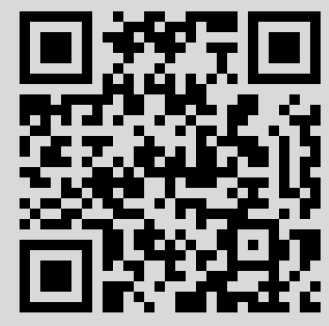




\title{
СЕДЛОВАЯ ТОЧКА ДЛЯ ДИФФЕРЕНЦИАЛЬНЫХ ИГР С СИЛЬНО ВЫПУКЛО-ВОГНУТЫМ ИНТЕГРАНТОМ
}

\section{Г.Е. Иванов}

\begin{abstract}
В работе рассматриваются нелинейные дифференциальные игры с нулевой суммой на фиксированном отрезке времени, для которых подынтегральная функция (интегрант) в функционале качества является достаточно сильно выпукло-вогнутой функцией по управлениям игроков. Показано, что в рассматриваемой постановке существует седловая точка в классе программных стратегий и принцип минимакса, аналогичный принципу максимума Понтрягина, является необходимым и достаточным условием оптимальности. Рассмотрен пример, на основе которого проведено сопоставление исследуемого класса игр с двумя известными классами дифференциальных игр.

Библиографоял: 7 названий.
\end{abstract}

В теории дифференциальных игр с нулевой суммой рассматривается динамическая система, на которую воздействуют два игрока, имеющие противоположные цели. Общий метод определения оптимальных стратегий игроков [1]-[3] основан на конструкции стабильного моста, которая обобщает метод динамического программирования в оптимальном управлении на задачи теории дифференциальных игр. Методы решения задач оптимального управления можно условно разделить на методы, основанные на принципе динамического программирования Р. Беллмана и методы, основанные на принципе максимума Л. С. Понтрягина. Методы первой групшы являются глобальными в том смысле, что они решают задачу оптимального управления сразу для всех значений фазового вектора. Методы второй группы - локальные, они позволяют находить оптимальное управление и оптимальную фазовую траекторию исключительно для заданного начального значения фазового вектора. Естественно, вычислительные алгоритмы, основанные на локальных методах, как правило эффективнее глобальных.

В теории дифференциальных игр локальные методы в общем случае не применимы, так как оптимальное программное управление (программное управление - управление, зависящее от времени и начальной позиции, но не зависящее от текущего значения фазового вектора) одного игрока зависит от управления другого игрока. Если в дифференциальной игре существует седловая точка в классе программных стратегий, то для каждого игрока $A$ существует единая программная стратегия такая, что при любых допустимых действиях другого игрока $B$ игроку $A$ гарантировано некоторое значение

Работа выполнена при финансовой поддержке Российского фонда фундаментальных исследований, грант № 95-01-00771a. 
функционала качества, которое не может быть улучшено за счет выбора другой (необязательно программной) стратегии игрока $A$. Программные стратегии игроков, образующие седловую точку, естественно назвать оптимальными программными управлениями, а траекторию фазового вектора, получающуюся при таких управлениях, оптимальной траекторией. В этом случае решение дифференциальной игры может быть основано на принципе максимума Понтрягина.

В настоящей работе рассматриваются нелинейные дифференциальные игры с нулевой суммой на фиксированном отрезке времени, функционал качества является суммой интегрального и терминального слагаемых. Основное требование состоит в том, чтобы интегрант (подьнтегральная функция в интегральном слагаемом функционала качества) был сильно выпукльм по управлению игрока, минимизирующего функционал качества, и сильно вогнутым по управлению игрока-максимизирующего. Причем соответствующие константы сильной вьпуклости и вогнутости должны быть не меньше некоторого выражения, зависящего от констант гладкости функций, определяющих правую часть системы дифференциальных уравнений и функционал качества игры. В работе показано, что при этих условиях существует седловая точка в классепрограммных стратегий и принцип минимакса, аналогичный принципу максимума Понтрягина, является необходимым и достаточньм условием оптимальности.

Рассмотрен пример, на основе которого проведено сопоставление исследуемого класса игр с двумя известными классами дифференциальных игр: классом линейно-квадратичных игр и классом игр с чисто геометрическими ограничениями на управления игроков.

1. Постановка задачи. Рассмотрим дифференциальную игру

$$
\dot{x}(t)=f(t, x(t), u(t), v(t)), \quad x\left(t_{0}\right)=x_{0}
$$

на отрезке времени $t \in\left[t_{0}, T\right], 0 \leqslant t_{0}<T$, с функционалом качества

$$
\mathscr{J}=\gamma(x(T))+\int_{t_{0}}^{T} \zeta(t, x(t), u(t), v(t)) d t
$$

где $u(t) \in P(t) \subset \mathbb{R}^{p}$ - управление первого игрока, стремящегося минимизировать функционал $\mathscr{J}, v(t) \in Q(t) \subset \mathbb{R}^{q}$ - управление второго игрока, максимизирующего $\mathscr{J}, x(t) \in \mathbb{R}^{n}-$ фазовьй вектор системы;

$$
f:[0, T] \times \mathbb{R}^{n} \times \mathbb{R}^{p} \times \mathbb{R}^{q} \rightarrow \mathbb{R}^{n}, \quad \gamma: \mathbb{R}^{n} \rightarrow \mathbb{R}, \quad \zeta:[0, T] \times \mathbb{R}^{n} \times \mathbb{R}^{p} \times \mathbb{R}^{q} \rightarrow \overline{\mathbb{R}}
$$

- заданные функции.

Через $\mathbb{R}^{s}$ обозначено пространство $s$-мерных векторов-столбцов, через $\overline{\mathbb{R}}$ - расширенная числовая прямая: $\overline{\mathbb{R}}=\mathbb{R} \cup\{+\infty,-\infty\}$.

Будем предполагать, что для любых $t \in[0, T], x \in \mathbb{R}^{n}$

$$
\begin{array}{llll}
\zeta(t, x, u, v)=+\infty & \text { при } & u \in \mathbb{R}^{p} \backslash P(t), \quad v \in Q(t), \\
\zeta(t, x, u, v)=-\infty & \text { при } & u \in P(t), \quad v \in \mathbb{R}^{q} \backslash Q(t), \\
\zeta(t, x, u, v) \in \mathbb{R} & \text { при } & u \in P(t), \quad v \in Q(t),
\end{array}
$$

что автоматически учитывает ограничения $u(t) \in P(t), v(t) \in Q(t)$. 
Через $\mathscr{L}_{s}^{m}$ будем обозначать пространство суммируемых в $m$-й степени (относительно лебеговой меры) функций $\varphi:\left[t_{0}, T\right] \rightarrow \mathbb{R}^{s}$ (если $t_{0}$ не определено, то предполагаем $\left.t_{0}=0\right)$.

Предполагаем далее, что для любых $x \in \mathbb{R}^{n}, u \in \mathbb{R}^{p}, v \in \mathbb{R}^{q}$ функция $f(\cdot, x, u, v)$ измерима на $[0, T]$; для любого $t \in[0, T]$ определено непустое выпуклое открытое множество $X(t)$. Для любого $t \in[0, T]$ функция $f(t, \cdot)$ дифференцируема на множестве $X(t) \times P(t) \times Q(t)$ и вьполнено условие продолжимости решений, т.е. для любых $t_{0} \in[0, T), x_{0} \in X\left(t_{0}\right), u \in \mathscr{L}_{p}^{2}, v \in \mathscr{L}_{q}^{2}$ таких, что $u(t) \in P(t), v(t) \in Q(t)$ при $t \in\left[t_{0}, T\right]$, сушествует решение в смысле Каратеодори задачи Коши $(1.1)$, причем $x(t) \in X(t)$ для любого $t \in\left[t_{0}, T\right]$. Обозначим это решение через $\chi\left(t, t_{0}, x_{0}, u, v\right)$.

Договоримся, что при дифференцировании $n$-мерного столбца по $m$-мерному столбцу получается $n \times m$-матрища, при дифференцировании $n$-мерной строки по $m$-мерной строке получается $m \times n$-матрица.

Будем говорить, что матрица-функция $M(t) \in \mathbb{R}^{n \times m}$ удовлетворяет условию Липшица с константой $L$ на множестве $\mathscr{T}$, если

$$
\forall t_{1}, t_{2} \in \mathscr{T} \quad\left\|M\left(t_{1}\right)-M\left(t_{2}\right)\right\| \leqslant L\left|t_{1}-t_{2}\right|
$$

Будем предполагать далее, что для любых $t \in[0, T]$ на множестве $X(t) \times P(t) \times Q(t)$ матрица производных $\partial f(t, x, u, v) / \partial x$ ограничена по норме числом $N_{x}^{f}(t)$ и удовлетворяет условию Липшица по $x, u, v$ с константами $L_{x x}^{f}(t), L_{x u}^{f}(t), L_{x v}^{f}(t)$; матрица производных $\partial f(t, x, u, v) / \partial u$ ограничена по норме числом $N_{u}^{f}(t)$ и удовлетворяет условию Липшица по $u$ с константой $L_{u u}^{f}(t)$; матрица производных $\partial f(t, x, u, v) / \partial v$ ограничена по норме числом $N_{v}^{f}(t)$ и удовлетворяет условию Липшица по $v$ с константой $L_{v v}^{f}(t)$; кроме того,

$$
|f(t, x, u, v)| \leqslant M^{f}(t)+N_{x}^{f}(t)|x|+N_{u}^{f}(t)|u|+N_{v}^{f}(t)|v| .
$$

ОПРЕДЕЛЕНИЕ. Пусть $G \subset \mathbb{R}^{s}$ - выпуклое множество. Будем говорить, что собственная функция $g: \mathbb{R}^{s} \rightarrow \overline{\mathbb{R}}$ слабо выпукла на $G$ с константой $R$, если функция $x \mapsto$ $g(x)+\frac{1}{2} R|x|^{2}$ выпукла на $G$.

Функция $g$ называется сильно выпуклой $c$ константой $R>0$, если она слабо выпукла с константой $-R$ на $\mathbb{R}^{s}$.

Функция $g$ назьвается слабо (сильно) вогнутой с константой $R$, если $-g$ слабо (сильно) вьпукла с той же константой $R$.

Предполагаем далее, что функция $\gamma$ дифференцируема, слабо выпукла с константой $R^{+\gamma} \geqslant 0$ и слабо вогнута с константой $R^{-\gamma} \geqslant 0$ на множестве $X(T)$. Определим константу $N^{\gamma} \in \mathbb{R} \cup\{+\infty\}$ :

$$
N^{\gamma}=\sup _{x \in X(T)}\left|\frac{d \gamma(x)}{d x}\right|
$$

Будем предполагать далее, что функция $\zeta:[0, T] \times \mathbb{R}^{n} \times \mathbb{R}^{p} \times \mathbb{R}^{q} \rightarrow \overline{\mathbb{R}}$ борелевская; для любых $t \in[0, T], x \in X(t), u \in P(t), v \in Q(t)$ функция $\zeta(t, x, u, v)$ сильно вьпукла с константой $R_{u}^{\zeta}(t)$ и полунепрерывна снизу по $u \in \mathbb{R}^{p}$, сильно вогнута с константой $R_{v}^{\zeta}(t)$ и полунепрерывна сверху по $v \in \mathbb{R}^{q}$, причем

$$
R_{u}^{\zeta}(t) \geqslant R_{u, \min }^{\zeta}>0, \quad R_{v}^{\zeta}(t) \geqslant R_{v, \min }^{\zeta}>0
$$


для любых $t \in[0, T], u \in P(t), v \in Q(t)$ функция $\zeta(t, \cdot, u, v)$ дифференцируема на $X(t)$, градиент $\partial \zeta(t, x, u, v) / \partial x$ удовлетворяет условию Липшица по $u, v$ с константами $L_{x u}^{\zeta}(t)$, $L_{x v}^{\zeta}(t) ;$ функция $\zeta$ слабо вьпукла и слабо вогнута по $x$ с константами $R_{x}^{+\zeta}(t), R_{x}^{-\zeta}(t) \geqslant 0$ на $X(t)$;

$$
\begin{aligned}
& \forall t \in[0, T], \quad x \in X(t), \quad u \in P(t), \quad v \in Q(t) \\
& \quad\left\|\frac{\partial \zeta(t, x, u, v)}{\partial x}\right\| \leqslant M_{x}^{\zeta}(t)+L_{x x}^{\zeta}(t)|x|+L_{x u}^{\zeta}(t)|u|+L_{x v}^{\zeta}(t)|v|
\end{aligned}
$$

Определим функцию $N_{x}^{\zeta}:[0, T] \rightarrow \mathbb{R} \cup\{+\infty\}$ :

$$
N_{x}^{\zeta}(t)=\sup _{x \in X(t), u \in P(t), v \in Q(t)}\left\|\frac{\partial \zeta(t, x, u, v)}{\partial x}\right\| .
$$

Пусть $t \in[0, T], x \in X(t), u \in P(t), v \in Q(t)$. Определим

$$
u_{0}(t, x, v)=\underset{u \in \mathbb{R}^{p}}{\arg \min } \zeta(t, x, u, v), \quad v_{0}(t, x, u)=\underset{v \in \mathbb{R}^{q}}{\arg \max } \zeta(t, x, u, v) .
$$

Будем предполагать, что для любых $t \in[0, T], x \in X(t), u \in P(t), v \in Q(t)$

$\left|u_{0}(t, x, v)\right| \leqslant M^{u}(t)+M_{v}^{u}(t)|v|+M_{x}^{u}(t)|x|, \quad\left|v_{0}(t, x, u)\right| \leqslant M^{v}(t)+M_{u}^{v}(t)|u|+M_{x}^{v}(t)|x|$.

Зафиксируем произвольную непрерьвную функцию $x^{0}:[0, T] \rightarrow \mathbb{R}^{n}$ такую, что $x^{0}(t) \in X(t)$ для любого $t \in[0, T]$. Обозначим через $u_{s}^{0}(t), v_{s}^{0}(t)$ седловую точку функции $\zeta\left(t, x^{0}(t), \cdot, \cdot\right)$ :

$\forall u \in \mathbb{R}^{p}, \quad v \in \mathbb{R}^{q} \quad \zeta\left(t, x^{0}(t), u_{s}^{0}(t), v\right) \leqslant \zeta\left(t, x^{0}(t), u_{s}^{0}(t), v_{s}^{0}(t)\right) \leqslant \zeta\left(t, x^{0}(t), u, v_{s}^{0}(t)\right)$.

Будем предполагать, что функция $t \rightarrow \zeta\left(t, x^{0}(t), u_{s}^{0}(t), v_{s}^{0}(t)\right)$ принадлежит класcy $\mathscr{L}_{1}^{1}$, а также $u_{s}^{0} \in \mathscr{L}_{p}^{2}, v_{s}^{0} \in \mathscr{L}_{q}^{2}$,

$$
\begin{gathered}
M^{f}, N_{x}^{f}, L_{x x}^{f}, R_{x}^{+\zeta}, R_{x}^{-\zeta}, M_{x}^{\zeta}, L_{x x}^{\zeta} \in \mathscr{L}_{1}^{1}, \\
N_{u}^{f}, N_{v}^{f}, L_{x u}^{f}, L_{x v}^{f}, L_{x u}^{\zeta}, L_{x v}^{\zeta}, M^{u}, M^{v}, M_{x}^{u}, M_{x}^{v} \in \mathscr{L}_{1}^{2}, \\
R_{u}^{\zeta}, R_{v}^{\zeta}, L_{u u}^{f}, L_{v v}^{f}, M_{v}^{u}, M_{u}^{v} \in \mathscr{L}_{1}^{\infty} .
\end{gathered}
$$

Определим множества допустимых стратегий первого и второго игроков:

$$
\begin{aligned}
& \mathscr{U}=\left\{u \in \mathscr{L}_{p}^{2}: \int_{t_{0}}^{T}\left(\max _{v \in \mathbb{R}^{q}} \zeta\left(t, x^{0}(t), u(t), v\right)\right) d t<+\infty\right\}, \\
& \mathscr{V}=\left\{v \in \mathscr{L}_{q}^{2}: \int_{t_{0}}^{T}\left(\min _{u \in \mathbb{R}^{p}} \zeta\left(t, x^{0}(t), u, v(t)\right)\right) d t>-\infty\right\} .
\end{aligned}
$$

Заметим, что $u_{s}^{0} \in \mathscr{U}, v_{s}^{0} \in \mathscr{V}$, следовательно, множества допустимых стратегий непусты. В силу (1.5) множества $\mathscr{U}, \mathscr{V}$ не зависят от выбора непрерывной функции $x^{0}(t) \in X(t)$. 
Определим функцию

$$
e(t)=\exp \left(\int_{t}^{T} N_{x}^{f}(\tau) d \tau\right)
$$

и числа

$$
\begin{gathered}
K_{u u}^{f}=\underset{t \in[0, T]}{\operatorname{ess} \sup } \frac{L_{u u}^{f}(t) e(t)}{R_{u}^{\zeta}(t)}, \quad K_{v v}^{f}=\underset{t \in[0, T]}{\operatorname{ess} \sup } \frac{L_{v v}^{f}(t) e(t)}{R_{v}^{\zeta}(t)}, \\
K_{u}^{f}=\int_{0}^{T} \frac{\left(N_{u}^{f}(t) e(t)\right)^{2}}{R_{u}^{\zeta}(t)} d t, \quad K_{v}^{f}=\int_{0}^{T} \frac{\left(N_{v}^{f}(t) e(t)\right)^{2}}{R_{v}^{\zeta}(t)} d t, \\
K_{x u}^{f}=\int_{0}^{T} \frac{\left(L_{x u}^{f}(t) e(t)\right)^{2}}{R_{u}^{\zeta}(t)} d t, \quad K_{x v}^{f}=\int_{0}^{T} \frac{\left(L_{x v}^{f}(t) e(t)\right)^{2}}{R_{v}^{\zeta}(t)} d t, \\
K_{x u}^{\zeta}=\int_{0}^{T} \frac{\left(L_{x u}^{\zeta}(t)\right)^{2}}{R_{u}^{\zeta}(t)} d t, \quad K_{x v}^{\zeta}=\int_{0}^{T} \frac{\left(L_{x v}^{\zeta}(t)\right)^{2}}{R_{v}^{\zeta}(t)} d t, \\
K_{x x}^{f}=\int_{0}^{T} L_{x x}^{f}(t) e(t) d t, \quad K_{x x}^{+\zeta}=\int_{0}^{T} R_{x}^{+\zeta}(t) d t, \quad K_{x x}^{-\zeta}=\int_{0}^{T} R_{x}^{-\zeta}(t) d t,
\end{gathered}
$$

определим константу $K^{\gamma} \in \mathbb{R} \cup\{+\infty\}$ :

$$
K^{\gamma}=N^{\gamma}+\int_{0}^{T} N_{x}^{\zeta}(t) d t
$$

Будем предполагать, что либо динамика системы (1.1) линейна:

$$
f(t, x, u, v)=A(t) x+B(t) u+C(t) v
$$

либо $K^{\gamma}<+\infty$.

Определим константы

$$
\begin{aligned}
& K_{u u}^{J}=K^{\gamma}\left(K_{u u}^{f}+2\left(K_{x u}^{f} K_{u}^{f}\right)^{1 / 2}+K_{x x}^{f} K_{u}^{f}\right)+2\left(K_{x u}^{\zeta} K_{u}^{f}\right)^{1 / 2}+K_{x x}^{+\zeta} K_{u}^{f}+R^{+\gamma} K_{u}^{f}, \\
& K_{v v}^{J}=K^{\gamma}\left(K_{v v}^{f}+2\left(K_{x v}^{f} K_{v}^{f}\right)^{1 / 2}+K_{x x}^{f} K_{v}^{f}\right)+2\left(K_{x v}^{\zeta} K_{v}^{f}\right)^{1 / 2}+K_{x x}^{-\zeta} K_{v}^{f}+R^{-\gamma} K_{v}^{f} .
\end{aligned}
$$

Положим по определению $+\infty \cdot 0=0 \cdot(+\infty)=0$. Поэтому в случае линейной динамики (1.10) первые слагаемые в (1.11) равны 0 даже в том случае, когда $K^{\gamma}=+\infty$.

Наконец, будем предполагать, что

$$
K_{u u}^{J}<1, \quad K_{v v}^{J}<1
$$

Заметим, что для любых функций $N_{x}^{f}, N_{u}^{f}, N_{v}^{f}, L_{x x}^{f}, L_{x u}^{f}, L_{x v}^{f}, L_{u u}^{f}, L_{v v}^{f}, L_{x u}^{\zeta}, L_{x v}^{\zeta}$, $R_{x}^{+\zeta}, R_{x}^{-\zeta}$ классов (1.8) найдутся достаточно большие числа $R_{u, \min }^{\zeta}, R_{v, \min }^{\zeta}(1.4)$ такие, что вьполнены неравенства (1.12). 
2. Вспомогательные результаты. Через $\mathbb{R}^{s *}$ будем обозначать пространство $s$-мерных вектор-строк, через $\mathscr{L}_{s *}^{m}$ - пространство суммируемых в $m$-й степени функций $\varphi:\left[t_{0}, T\right] \rightarrow \mathbb{R}^{s *}$.

Пусть собственная функция $g: \mathbb{R}^{s} \rightarrow \overline{\mathbb{R}}$ дифференцируема в точке $\bar{x} \in \mathbb{R}^{n}$ по любому направлению, т.е.

$$
\forall y \in \mathbb{R}^{n} \quad \exists g^{\prime}(\bar{x}, y)=\lim _{t \rightarrow+0} \frac{1}{t}(g(\bar{x}+t y)-g(\bar{x})) \in \overline{\mathbb{R}}
$$

тогда субдифференциалом функции $g$ в точке $\bar{x}$ назьвается множество

$$
\partial g(\bar{x})=\left\{\xi \in \mathbb{R}^{n *}: \xi y \leqslant g^{\prime}(\bar{x}, y) \quad \forall y \in \mathbb{R}^{n}\right\}
$$

В дальнейшем будем использовать следующую простую лемму.

Лемма 1. Пусть собственная функиия $g: \mathbb{R}^{n} \rightarrow \overline{\mathbb{R}}$ слабо выпукла с константой $R$ на выпуклом множестве $X \subset \mathbb{R}^{n}, \bar{x} \in X, \xi \in \partial g(\bar{x})$. Тогда

$$
\forall x \in X \quad g(x) \geqslant g(\bar{x})+\xi(x-\bar{x})-\frac{1}{2} R|x-\bar{x}|^{2}
$$

ДокАЗАТЕЛЬСтво. Определим функцию

$$
g_{0}(x)=g(x)-g(\bar{x})-\xi(x-\bar{x})+\frac{1}{2} R|x-\bar{x}|^{2} .
$$

Заметим, что функция $g_{0}$ выпукла и $0 \in \partial g_{0}(\bar{x})$, следовательно, для любого $x \in X$ $g_{0}(x) \geqslant g_{0}(\bar{x})=0$.

Пусть $t_{0} \in[0, T], x_{0} \in X\left(t_{0}\right), u \in \mathscr{U}, v \in \mathscr{V}$. Определим функционал качества игры:

$$
\mathscr{J}\left(t_{0}, x_{0}, u, v\right)=\gamma\left(\chi\left(T, t_{0}, x_{0}, u, v\right)\right)+\int_{t_{0}}^{T} \zeta\left(t, \chi\left(t, t_{0}, x_{0}, u, v\right), u(t), v(t)\right) d t
$$

Определим вспомогательный функционал $\rho: \mathscr{L}_{p}^{2} \rightarrow \mathbb{R}$, который в силу неравенства (1.4) и включения $R_{u}^{\zeta} \in \mathscr{L}_{1}^{\infty}$ эквивалентен норме пространства $\mathscr{L}_{p}^{2}$ :

$$
\rho(u)=\left(\int_{t_{0}}^{T} R_{u}^{\zeta}(t)|u(t)|^{2} d t\right)^{1 / 2}
$$

Зафиксируем произвольные $t_{0} \in[0, T), x_{0} \in X\left(t_{0}\right), \bar{u} \in \mathscr{U}, \bar{v} \in \mathscr{V}$.

Введем обозначения

$$
\begin{gathered}
\bar{x}(t)=\chi\left(t, t_{0}, x_{0}, \bar{u}, \bar{v}\right), \quad \zeta_{x}(t)=\frac{\partial \zeta}{\partial x}(t, \bar{x}(t), \bar{u}(t), \bar{v}(t)) \\
f_{x}(t)=\frac{\partial f}{\partial x}(t, \bar{x}(t), \bar{u}(t), \bar{v}(t)), \quad f_{u}(t)=\frac{\partial f}{\partial u}(t, \bar{x}(t), \bar{u}(t), \bar{v}(t)) .
\end{gathered}
$$


Для любых $\eta \in X\left(t_{0}\right), u \in \mathscr{U}$ определим

$$
\begin{aligned}
\Delta \mathscr{J}(\eta, u)= & \gamma\left(\chi\left(T, t_{0}, \eta, u, \bar{v}\right)-\gamma(\bar{x}(T))\right. \\
& +\int_{t_{0}}^{T}\left(\zeta\left(t, \chi\left(t, t_{0}, \eta, u, \bar{v}\right), u(t), \bar{v}(t)\right)-\zeta(t, \bar{x}(t), u(t), \bar{v}(t))\right) d t
\end{aligned}
$$

Определим $n \times n$-матрицу $\Phi(t, \tau)$ как решение линейной системы

$$
\frac{\partial \Phi(t, \tau)}{\partial t}=-\Phi(t, \tau) f_{x}(t)
$$

с начальньп условием $\Phi(\tau, \tau)=E$.

Определим строку $d \in \mathbb{R}^{n *}$ :

$$
d=\frac{d \gamma}{d x}(\bar{x}(T)) \Phi\left(t_{0}, T\right)+\int_{t_{0}}^{T} \zeta_{x}(\tau) \Phi\left(t_{0}, \tau\right) d \tau
$$

функцию-строку $\mathscr{D} \in \mathscr{L}_{p *}^{2}$ :

$$
\mathscr{D}(t)=\frac{d \gamma}{d x}(\bar{x}(T)) \Phi(t, T) f_{u}(t)+\left(\int_{t}^{T} \zeta_{x}(\tau) \Phi(t, \tau) d \tau\right) f_{u}(t),
$$

а также константы

$$
\begin{gathered}
K_{u \eta}^{J}=e(0)\left(K^{\gamma}\left(K_{x x}^{f}\left(K_{u}^{f}\right)^{1 / 2}+\left(K_{x u}^{f}\right)^{1 / 2}\right)+\left(R^{+\gamma}+K_{x x}^{+\zeta}\right)\left(K_{u}^{f}\right)^{1 / 2}+K_{x u}^{\zeta}\right) \\
K_{\eta \eta}^{+J}=(e(0))^{2}\left(K^{\gamma} K_{x x}^{f}+R^{+\gamma}+K_{x x}^{+\zeta}\right) .
\end{gathered}
$$

Для доказательства вьпуклости и полунепрерьвности снизу функционала (2.1) по $u \in \mathscr{U}$ понадобится следующая лемма.

ЛЕмма 2. Для любых $\eta \in X\left(t_{0}\right), u \in \mathscr{U}$ выполнено неравенство

$$
\begin{aligned}
\Delta \mathscr{J}(\eta, u) \geqslant & \int_{t_{0}}^{T} \mathscr{D}(t) \Delta u(t) d t+d \Delta \eta \\
& \quad-\frac{1}{2} K_{u u}^{J} \rho^{2}(\Delta u)-K_{u \eta}^{J} \rho(\Delta u)|\Delta \eta|-\frac{1}{2} K_{\eta \eta}^{+J}|\Delta \eta|^{2},
\end{aligned}
$$

әде $\Delta u(t)=u(t)-\bar{u}(t), \Delta \eta=\eta-x_{0}$.

ДокАЗАТЕЛЬСТво. Введем обозначения

$$
x(t)=\chi\left(t, t_{0}, \eta, u, \bar{v}\right), \quad \Delta x(t)=x(t)-\bar{x}(t) .
$$

Заметим, что

$$
\frac{d}{d t} \Delta x(t)=f(t, x(t), u(t), \bar{v}(t))-f(t, \bar{x}(t), \bar{u}(t), \bar{v}(t)),
$$

следовательно,

$$
\frac{d}{d t}|x(t)| \leqslant N_{x}^{f}(t)|\Delta x(t)|+N_{u}^{f}(t)|\Delta u(t)|,
$$


поэтому $|\Delta x(t)| \leqslant \widehat{\Delta x}$, где

$$
\widehat{\Delta x}=|\Delta \eta| e(0)+\left(K_{u}^{f}\right)^{1 / 2} \rho(\Delta u) .
$$

Кроме того,

$$
\begin{gathered}
\left|\frac{d}{d t} \Delta x(t)-f_{x}(t) \Delta x(t)-f_{u}(t) \Delta u(t)\right| \\
\leqslant \frac{1}{2} L_{x x}^{f}(t) \widehat{(\Delta x)}^{2}+L_{x u}^{f}(t) \widehat{\Delta x}|\Delta u(t)|+\frac{1}{2} L_{u u}^{f}(t)|\Delta u(t)|^{2} \\
\|\Phi(t, \tau)\| \leqslant e(t) \quad \text { при } 0 \leqslant t \leqslant \tau \leqslant T .
\end{gathered}
$$

Следовательно,

$$
\begin{aligned}
& \left|\frac{d}{d t}(\Phi(t, \tau) \Delta x(t))-\Phi(t, \tau) f_{u}(t) \Delta u(t)\right| \\
& \quad \leqslant e(t)\left(\frac{1}{2} L_{x x}^{f}(t) \widehat{(\Delta x)^{2}}+L_{x u}^{f}\left(\left.t \widehat{\Delta x \mid} \Delta u(t)\left|+\frac{1}{2} L_{u u}^{f}(t)\right| \Delta u(t)\right|^{2}\right),\right.
\end{aligned}
$$

откуда с учетом $\Phi(\tau, \tau)=E$ получаем

$$
\left|\Delta x(\tau)-\Phi\left(t_{0}, \tau\right) \Delta \eta-\int_{t_{0}}^{\tau} \Phi(\xi, \tau) f_{u}(\xi) \Delta u(\xi) d \xi\right| \leqslant K_{1}
$$

где

$$
K_{1}=\frac{1}{2} K_{x x}^{f} \widehat{(\Delta x)}^{2}+\left(K_{x u}^{f}\right)^{1 / 2} \widehat{\Delta x} \rho(\Delta u)+\frac{1}{2} K_{u u}^{f} \rho^{2}(\Delta u) .
$$

Заметим, что в силу леммы 1

$$
\begin{aligned}
& \zeta(t, x(t), u(t), \bar{v}(t))-\zeta(t, \bar{x}(t), u(t), \bar{v}(t)) \\
& \geqslant \zeta_{x}(t) \Delta x(t)-\frac{1}{2} R_{x}^{+\zeta}(t)|\Delta x(t)|^{2}-L_{x u}^{\zeta}(t)|\Delta x(t)| \cdot|\Delta u(t)| \\
& \gamma(x(T))-\gamma(\bar{x}(T)) \geqslant \frac{d \gamma}{d x}(\bar{x}(T)) \Delta x(T)-\frac{1}{2} R^{+\gamma}|\Delta x(T)|^{2}
\end{aligned}
$$

откуда и из (2.4) получаем

$$
\Delta \mathscr{J}(\eta, u) \geqslant \int_{t_{0}}^{T} \zeta_{x}(\tau) \Delta x(\tau) d \tau+\frac{d \gamma}{d x}(\bar{x}(T)) \Delta x(T)-K_{2}
$$

где

$$
K_{2}=\frac{1}{2} R^{+\gamma}{\widehat{(\Delta x)^{2}}}^{2}+\frac{1}{2} K_{x x}^{+\zeta} \widehat{(\Delta x)}^{2}+\left(K_{x u}^{\zeta}\right)^{1 / 2} \widehat{\Delta x} \rho(\Delta u) .
$$

Заметим, что по теореме Фубини

$$
\int_{t_{0}}^{T} \zeta_{x}(\tau)\left(\int_{t_{0}}^{\tau} \Phi(\xi, \tau) f_{u}(\xi) \Delta u(\xi) d \xi\right) d \tau=\int_{t_{0}}^{T}\left(\int_{t}^{T} \zeta_{x}(\tau) \Phi(t, \tau) d \tau\right) f_{u}(t) \Delta u(t) d t
$$


откуда и из $(2.5),(2.6),(2.9),(2.11)$ получаем

$$
\begin{aligned}
\Delta \mathscr{J}(\eta, u) \geqslant & \int_{t_{0}}^{T}\left(\int_{t}^{T} \zeta_{x}(\tau) \Phi(t, \tau) d \tau\right) f_{u}(t) \Delta u(t) d t+\left(\int_{t_{0}}^{T} \zeta_{x}(t) \Phi\left(t_{0}, t\right) d t\right) \Delta \eta \\
& +\frac{d \gamma}{d x}(\bar{x}(T))\left(\Phi\left(t_{0}, T\right) \Delta \eta+\int_{t_{0}}^{T} \Phi(t, T) f_{u}(t) \Delta u(t) d t\right)-K^{\gamma} K_{1}-K_{2} \\
= & \int_{t_{0}}^{T} \mathscr{D}(t) \Delta u(t) d t+d \Delta \eta-K^{\gamma} K_{1}-K_{2},
\end{aligned}
$$

причем в случае линейной динамики (1.10) $K_{1}=0$, и даже в случае $K^{\gamma}=+\infty$ следует положить $K^{\gamma} K_{1}=0$, что оправдьвает соглашение $+\infty \cdot 0=0$.

Последнее неравенство с учетом (1.11), (2.7), (2.8), (2.10), (2.12) дает требуемое утверждение.

Лемма 3. Для любых $t_{0} \in[0, T], x_{0} \in X\left(t_{0}\right), \bar{v} \in \mathscr{V}$ функиионал $\mathscr{J}\left(t_{0}, x_{0}, \cdot, \bar{v}\right)$, определенный (2.1), полунепрерывен снизу в смысле слабой топологии пространства $\mathscr{L}_{p}^{2}$.

ДокАЗАТЕЛЬСТво. Пусть $\left\{u_{i}\right\}$ - последовательность функций из $\mathscr{L}_{p}^{2}$, слабо в $\mathscr{L}_{p}^{2}$ сходящаяся к функции $\bar{u} \in \mathscr{L}_{p}^{2}$.

Из формул $(2.1),(2.4)$ следует, что

$$
\begin{aligned}
& \mathscr{J}\left(t_{0}, x_{0}, u_{i}, \bar{v}\right)-\mathscr{J}\left(t_{0}, x_{0}, \bar{u}, \bar{v}\right) \\
& \quad=\Delta \mathscr{J}\left(x_{0}, u_{i}\right)+\int_{t_{0}}^{T}\left(\zeta\left(t, \bar{x}(t), u_{i}(t), \bar{v}(t)\right)-\zeta(t, \bar{x}(t), \bar{u}(t), \bar{v}(t))\right) d t .
\end{aligned}
$$

В силу леммы 2, неравенства (1.12), формулы $(2.2)$ и включений $\mathscr{D}, \bar{u}^{T} \in \mathscr{L}_{p *}^{2}, R_{u}^{\zeta} \in$ $\mathscr{L}_{1}^{\infty}$ имеем

$$
\Delta \mathscr{J}\left(x_{0}, u_{i}\right)+\frac{1}{2} \rho^{2}\left(u_{i}\right)-\frac{1}{2} \rho^{2}(\bar{u}) \geqslant \int_{t_{0}}^{T}\left(\mathscr{D}(t)+R_{u}^{\zeta}(t) \bar{u}^{T}(t)\right)\left(u_{i}(t)-\bar{u}(t)\right) d t \stackrel{i \rightarrow \infty}{\longrightarrow} 0 .
$$

Определим функцию

$$
\varphi(t, u)=\zeta(t, \bar{x}(t), u, \bar{v}(t))-\frac{1}{2} R_{u}^{\zeta}(t)|u|^{2}
$$

В силу принятых предположений о функции $\zeta$ функция $\varphi$ является вьпукльм нормальным интегрантом [4], [5].

Пусть функция $u_{0}(t, x, v)$ определена формулой (1.6). Определим

$$
\bar{u}_{0}(t)=u_{0}(t, \bar{x}(t), \bar{v}(t)), \quad \xi(t)=-R_{u}^{\zeta}(t) \bar{u}_{0}^{T}(t) .
$$

Тогда в силу $(1.3),(1.7),(1.9) \bar{u}_{0} \in \mathscr{L}_{p}^{2}, \xi \in \mathscr{L}_{p *}^{2}, \xi(t) \in \partial_{u} \varphi\left(t, \bar{u}_{0}(t)\right)$, следовательно,

$$
\begin{aligned}
\varphi^{*}(t, \xi(t)) & =\sup _{u \in \mathbb{R}^{p}}(\xi(t) u-\varphi(t, u))=\xi(t) \bar{u}_{0}(t)-\varphi\left(t, \bar{u}_{0}(t)\right) \\
& =-R_{u}^{\zeta}(t)\left|\bar{u}_{0}(t)\right|^{2}-\varphi\left(t, \bar{u}_{0}(t)\right) \leqslant-\zeta\left(t, \bar{x}(t), \bar{u}_{0}(t), \bar{v}(t)\right)
\end{aligned}
$$


Заметим, что из условия $\bar{v} \in \mathscr{V}$ и из определения множества $\mathscr{V}(1.9)$ следует

$$
\int_{t_{0}}^{T} \zeta\left(t, x^{0}(t), \bar{u}_{0}(t), \bar{v}(t)\right) d t>-\infty
$$

что в силу (1.5) и включений $\bar{x}-x^{0} \in \mathscr{L}_{n}^{\infty}, \bar{u} \in \mathscr{L}_{p}^{2}, M_{x}^{\zeta}, L_{x x}^{\zeta} \in \mathscr{L}_{1}^{1}, L_{x u}^{\zeta}, L_{x v}^{\zeta} \in \mathscr{L}_{1}^{2}$ дает

$$
\int_{t_{0}}^{T} \zeta\left(t, \bar{x}(t), \bar{u}_{0}(t), \bar{v}(t)\right) d t>-\infty
$$

откуда и из (2.16) получаем

$$
\int_{t_{0}}^{T} \varphi^{*}(t, \xi(t)) d t<+\infty
$$

При этих условиях, как показано в работе Рокафеллара [4, с. 188, следствие 3D], функционал

$$
u \rightarrow \int_{t_{0}}^{T} \varphi(t, u(t)) d t
$$

полунепрерьвен снизу в слабой топологии $\mathscr{L}_{p}^{2}$, следовательно,

$$
\varliminf_{i \rightarrow \infty} \int_{t_{0}}^{T}\left(\varphi\left(t, u_{i}(t)\right)-\varphi(t, \bar{u}(t))\right) d t \geqslant 0 .
$$

Откуда и из (2.13)-(2.15) получаем требуемое утверждение.

Лемма 4. Пусть $t_{0} \in[0, T], x_{0} \in X\left(t_{0}\right), \bar{v} \in \mathscr{V}, C \in \mathbb{R}$. Тогда лебегово множесmвo

$$
\mathscr{U}_{C}=\left\{u \in \mathscr{U}: \mathscr{J}\left(t_{0}, x_{0}, u, \bar{v}\right) \leqslant C\right\}
$$

либо пусто, либо предкомпактно в смысле слабой топологии пространства $\mathscr{L}_{p}^{2}$.

ДокАЗАТЕЛЬСтво. Пусть функция $u_{0}(t, x, v)$ определена формулой (1.6). Обозначим $\bar{f}(t, x)=f\left(t, x, u_{0}(t, x, \bar{v}(t)), \bar{v}(t)\right)$. Заметим, что в силу сильной выпуклости функции $\zeta$ по $u$ и липшицевости градиента $\partial \zeta(t, x, u, v) / \partial x$ по $u$ функция $u_{0}$ липшицева по $x$, а значит, и функция $\bar{f}$ липшицева по $x$. Кроме того, из условий $(1.3),(1.7)$ следует, что $|\bar{f}(t, x)| \leqslant C^{f}(t)+C_{x}^{f}(t)|x|$, где $C^{f}, C_{x}^{f} \in \mathscr{L}_{1}^{1}$.

Таким образом, существует решение задачи Коши $\dot{x}(t)=\bar{f}(t, x(t)), t \in\left[t_{0}, T\right]$, $x\left(t_{0}\right)=x_{0}$, которое мы обозначим $\bar{x}(t)$.

Определим $\bar{u}(t)=u_{0}(t, \bar{x}(t), \bar{v}(t))$. Применяя лемму 2, получим

$$
\Delta \mathscr{J}\left(x_{0}, u\right) \geqslant-K^{\mathscr{D}} \rho(\Delta u)-\frac{1}{2} K_{u u}^{J} \rho^{2}(\Delta u),
$$

где

$$
K^{\mathscr{D}}=\left(\int_{t_{0}}^{T} \frac{\mathscr{D}^{2}(t)}{R_{u}^{\zeta}(t)} d t\right)^{1 / 2}, \quad \Delta u=u-\bar{u} .
$$

Из леммы 1 с учетом условия $0 \in \partial_{u} \zeta(t, \bar{x}(t), \bar{u}(t), \bar{v}(t))$, получаем

$$
\zeta(t, \bar{x}(t), u(t), \bar{v}(t))-\zeta(t, \bar{x}(t), \bar{u}(t), \bar{v}(t)) \geqslant \frac{1}{2} R_{u}^{\zeta}(t)|u(t)-\bar{u}(t)|^{2},
$$


что с учетом $(2.1),(2.2),(2.4),(2.17)$ дает

$$
\mathscr{J}\left(t_{0}, x_{0}, u, \bar{v}\right)-\mathscr{J}\left(t_{0}, x_{0}, \bar{u}, \bar{v}\right) \geqslant \frac{1}{2}\left(1-K_{u u}^{J}\right) \rho^{2}(\Delta u)-K^{\mathscr{D}} \rho(\Delta u),
$$

следовательно,

$$
\rho(\Delta u) \leqslant \frac{K^{\mathscr{D}}}{1-K_{u u}^{J}}+\left(\left(\frac{K^{\mathscr{D}}}{1-K_{u u}^{J}}\right)^{2}+2 \frac{\mathscr{J}\left(t_{0}, x_{0}, u, \bar{v}\right)-\mathscr{J}\left(t_{0}, x_{0}, \bar{u}, \bar{v}\right)}{1-K_{u u}^{J}}\right)^{1 / 2},
$$

откуда с учетом (2.2) получаем, что если $u \in \mathscr{U}_{C}$, то

$$
\|u-\bar{u}\|_{\mathscr{L}_{p}^{2}} \leqslant \frac{1}{R_{u, \min }^{\zeta}}\left(\frac{K^{\mathscr{D}}}{1-K_{u u}^{J}}+\left(\left(\frac{K^{\mathscr{D}}}{1-K_{u u}^{J}}\right)^{2}+2 \frac{C-\mathscr{J}\left(t_{0}, x_{0}, \bar{u}, \bar{v}\right)}{1-K_{u u}^{J}}\right)^{1 / 2}\right),
$$

следовательно, множество $\mathscr{U}_{C}$ ограничено по норме $\mathscr{L}_{p}^{2}$ и (см. [6, с. 234]) предкомпактно в смысле слабой топологии пространства $\mathscr{L}_{p}^{2}$.

Пусть константы $K_{v v}^{J}, K_{u u}^{J}, K_{u \eta}^{J}, K_{\eta \eta}^{+J}$ определены формулами (1.11), (2.7), а константы $K_{v \eta}^{J}, K_{\eta \eta}^{-J}-$ формулами, аналогичными (2.7). Определим константы

$$
R_{x}^{+\Upsilon}=\frac{\left(K_{u \eta}^{J}\right)^{2}}{1-K_{u u}^{J}}+K_{\eta \eta}^{+J}, \quad R_{x}^{-\Upsilon}=\frac{\left(K_{v \eta}^{J}\right)^{2}}{1-K_{v v}^{J}}+K_{\eta \eta}^{-J} .
$$

Лемма 5. Для любых $\eta_{1}, \eta_{2} \in X\left(t_{0}\right), u_{1}, u_{2} \in \mathscr{U}, \bar{v} \in \mathscr{V}, \lambda \in[0,1]$ верно неравенство

$$
\delta \mathscr{J} \leqslant \frac{1}{2} \lambda(1-\lambda) R_{x}^{+\Upsilon}\left|\eta_{2}-\eta_{1}\right|^{2}
$$

əде

$$
\begin{gathered}
\delta \mathscr{J}=\mathscr{J}\left(t_{0}, x_{0}, \bar{u}, \bar{v}\right)-\lambda \mathscr{J}\left(t_{0}, \eta_{2}, u_{2}, \bar{v}\right)-(1-\lambda) \mathscr{J}\left(t_{0}, \eta_{1}, u_{1}, \bar{v}\right), \\
x_{0}=\lambda \eta_{1}+(1-\lambda) \eta_{2}, \quad \bar{u}(t)=\lambda u_{1}(t)+(1-\lambda) u_{2}(t) .
\end{gathered}
$$

ДокАЗАТЕЛЬСТво. Применяя лемму 2 для $\eta=\eta_{i}, u=u_{i}, i=1,2$, с учетом формул (2.1), (2.4) получим

$$
\begin{aligned}
\mathscr{J}\left(t_{0}, x_{0}, \bar{u}, \bar{v}\right)-\mathscr{J}\left(t_{0}, \eta_{i}, u_{i}, \bar{v}\right) \\
\leqslant \int_{t_{0}}^{T} \mathscr{D}(t)\left(\bar{u}(t)-u_{i}(t)\right) d t+d\left(x_{0}-\eta_{i}\right) \\
\quad+\int_{t_{0}}^{T}\left(\zeta(t, \bar{x}(t), \bar{u}(t), \bar{v}(t))-\zeta\left(t, \bar{x}(t), u_{i}(t), \bar{v}(t)\right)\right) d t \\
\quad-\frac{1}{2} K_{u u}^{J} \rho^{2}\left(\bar{u}-u_{i}\right)-K_{u \eta}^{J} \rho\left(\bar{u}-u_{i}\right)\left|x_{0}-\eta_{i}\right|-\frac{1}{2} K_{\eta \eta}^{+J}\left|x_{0}-\eta_{i}\right|^{2} .
\end{aligned}
$$

Складьвая эти неравенства с весами $\lambda$ и $1-\lambda$, с учетом сильной вьпуклости функции $\zeta$ по $u$ с константой $R_{u}^{\zeta}(t)$ и формулы $(2.2)$ получим

$$
\delta \mathscr{J} \leqslant \frac{\lambda(1-\lambda)}{2}\left(\left(K_{u u}^{J}-1\right) \rho^{2}\left(u_{2}-u_{1}\right)+2 K_{u \eta}^{J} \rho\left(u_{2}-u_{1}\right)\left|\eta_{2}-\eta_{1}\right|+K_{\eta \eta}^{+J}\left|\eta_{2}-\eta_{1}\right|^{2}\right) .
$$

Максимизируя правую часть последнего неравенства по $\rho\left(u_{2}-u_{1}\right)$ с учетом формул (1.12), (2.18), получим утверждение леммы. 


\section{3. Основные результаты.}

ТЕОремА 1. Для дифференциальной игры (1.1), (1.2) существует седловая точка в классе программных стратегий:

$$
\begin{gathered}
\forall t_{0} \in[0, T], \quad x_{0} \in X\left(t_{0}\right) \quad \exists \widehat{u} \in \mathscr{U}, \quad \widehat{v} \in \mathscr{V}: \quad \forall u \in \mathscr{U}, \quad v \in \mathscr{V} \\
\mathscr{J}\left(t_{0}, x_{0}, \widehat{u}, v\right) \leqslant \mathscr{J}\left(t_{0}, x_{0}, \widehat{u}, \widehat{v}\right) \leqslant \mathscr{J}\left(t_{0}, x_{0}, u, \widehat{v}\right) .
\end{gathered}
$$

ДокАЗАТЕЛьСтво. Из лемм $3-5$ следует, что функционал $\mathscr{J}\left(t_{0}, x_{0}, \cdot, v\right)$ вьпукльй, полунепрерьвньй снизу и его лебеговы множества $\mathscr{U}_{C}$ предкомпактны в смысле слабой топологии пространства $\mathscr{L}_{p}^{2}$. Аналогично, функционал $\mathscr{J}\left(t_{0}, x_{0}, u, \cdot\right)$ вогнут, полунепрерывен сверху и его лебеговы множества $\mathscr{V}_{C}=\left\{v \in \mathscr{V}: \mathscr{J}\left(t_{0}, x_{0}, u, v\right) \geqslant C\right\}$ предкомпактны в смысле слабой топологии пространства $\mathscr{L}_{q}^{2}$. Применяя теорему фон Неймана [7], получаем требуемое утверждение.

Теорема 1 показьвает, что для рассматриваемых дифференциальных игр, если один из игроков управляет оптимально, то другой игрок ничего не проиграет, если будет выбирать свое управление заранее на всем отрезке времени $\left[t_{0}, T\right]$, информация о состоянии фазового вектора системы в текущие моменты времени $t>t_{0}$ для него бесполезна. Однако, если один из игроков выберет неоптимальное управление, то другой игрок может этим воспользоваться, измерив значение фазового вектора в текущий момент времени и найдя оптимальное управление для дифференциальной игры (1.1), (1.2) на отрезке времени $[t, T]$.

Из теоремы 1 следует, что функция цены игры (см. [3, с. 78]) $\Upsilon$ может быть определена по следующей формуле:

$$
\Upsilon\left(t_{0}, x_{0}\right)=\min _{u \in \mathscr{U}} \max _{v \in \mathscr{V}} \mathscr{J}\left(t_{0}, x_{0}, u, v\right)=\max _{v \in \mathscr{V}} \min _{u \in \mathscr{U}} \mathscr{J}\left(t_{0}, x_{0}, u, v\right) .
$$

ТЕОрема 2. Функиия иены игры слабо выпукла с константой $R_{x}^{+\Upsilon}$ из (2.18) $u$ слабо вогнута с константой $R_{x}^{-\Upsilon}$ по начальной позиции $x_{0} \in X\left(t_{0}\right)$ для любого $t_{0} \in[0, T]$.

ДокАЗАтЕльство. Зафиксируем произвольные $t_{0} \in[0, T], \eta_{1}, \eta_{2} \in X\left(t_{0}\right), \lambda \in[0,1]$. Обозначим $\bar{\eta}=\lambda \eta_{1}+(1-\lambda) \eta_{2}$. В силу теоремы 1 существуют функция $\widehat{v} \in \mathscr{V}$ и функции $u_{i} \in \mathscr{U}, i=1,2$ :

$$
\Upsilon\left(t_{0}, \bar{\eta}\right)=\min _{u \in \mathscr{U}} \mathscr{J}\left(t_{0}, \bar{\eta}, u, \widehat{v}\right), \quad \Upsilon\left(t_{0}, \eta_{i}\right)=\max _{v \in \mathcal{V}} \mathscr{J}\left(t_{0}, \eta_{i}, u_{i}, v\right) .
$$

Определим $\bar{u}(t)=\lambda u_{1}(t)+(1-\lambda) u_{2}(t)$. Тогда из $(3.2)$ следует

$$
\Upsilon\left(t_{0}, \bar{\eta}\right) \leqslant \mathscr{J}\left(t_{0}, \bar{\eta}, \bar{u}, \widehat{v}\right), \quad \Upsilon\left(t_{0}, \eta_{i}\right) \geqslant \mathscr{J}\left(t_{0}, \eta_{i}, u_{i}, \widehat{v}\right) .
$$

Из леммы 5 получаем

$\lambda \mathscr{J}\left(t_{0}, \eta_{2}, u_{2}, \widehat{v}\right)+(1-\lambda) \mathscr{J}\left(t_{0}, \eta_{1}, u_{1}, \widehat{v}\right)-\mathscr{J}\left(t_{0}, \bar{\eta}, \bar{u}, \widehat{v}\right) \geqslant-\frac{1}{2} \lambda(1-\lambda) R_{x}^{+\Upsilon}\left|\eta_{2}-\eta_{1}\right|^{2}$, что вместе с (3.3) дает

$$
\lambda \Upsilon\left(t_{0}, \eta_{2}\right)+(1-\lambda) \Upsilon\left(t_{0}, \eta_{1}\right)-\Upsilon\left(t_{0}, \bar{\eta}\right) \geqslant-\frac{1}{2} \lambda(1-\lambda) R_{x}^{+\Upsilon}\left|\eta_{2}-\eta_{1}\right|^{2}
$$

Обобщенная вогнутость функции $\Upsilon\left(t_{0}, \cdot\right)$ доказывается аналогично.

Сформулируем принщип минимакса, аналогичньй принципу максимума Понтрягина. Определим функцию Понтрягина $\mathscr{H}:[0, T] \times \mathbb{R}^{n} \times \mathbb{R}^{p} \times \mathbb{R}^{q} \times \mathbb{R}^{n *} \rightarrow \overline{\mathbb{R}}$ :

$$
\mathscr{H}(t, x, u, v, \psi)=\psi f(t, x, u, v)+\zeta(t, x, u, v) .
$$


ТЕОрема 3. Для того чтобы управления $\widehat{u} \in \mathscr{U}, \widehat{v} \in \mathscr{V}$ были оптимальными, необходимо и достаточно, чтобы существовала абсолютно непрерывная функиия $\psi:\left[t_{0}, T\right] \rightarrow \mathbb{R}^{n *}$ такая, что почти всюду на отрезке $t \in\left[t_{0}, T\right]$ выполнено

$$
\begin{gathered}
\frac{d \psi(t)}{d t}=-\frac{\partial \mathscr{H}}{\partial x}(t, \widehat{x}(t), \widehat{u}(t), \widehat{v}(t), \psi(t)), \quad \psi(T)=\frac{d \gamma}{d x}(\widehat{x}(T)), \\
\frac{d \widehat{x}(t)}{d t}=\frac{\partial \mathscr{H}}{\partial \psi}(t, \widehat{x}(t), \widehat{u}(t), \widehat{v}(t), \psi(t)), \quad \widehat{x}\left(t_{0}\right)=x_{0}, \\
\mathscr{H}(t, \widehat{x}(t), \widehat{u}(t), v, \psi(t)) \leqslant \mathscr{H}(t, \widehat{x}(t), \widehat{u}(t), \widehat{v}(t), \psi(t)) \leqslant \mathscr{H}(t, \widehat{x}(t), u, \widehat{v}(t), \psi(t)) \\
\forall u \in \mathbb{R}^{p}, \quad v \in \mathbb{R}^{q} .
\end{gathered}
$$

ДокАЗАТЕЛЬСтво. Необходимость. Пусть $\widehat{u}(t), \widehat{v}(t)$ - оптимальные программные стратегии первого и второго игроков, $\widehat{x}(t)=\chi\left(t, t_{0}, x_{0}, \widehat{u}, \widehat{v}\right)-$ решение системы $(1.1)$ при этих управлениях, следовательно, имеет место (3.5). Как следует из теоремы 1 , оптимальное управление первого игрока $\widehat{u}(t)$ является решением задачи оптимального управления $(1.1),(1.2), J \rightarrow \min$, где в качестве управления $v(t)$ подставлено оптимальное управление $\widehat{v}(t)$. Пользуясь принципом максимума Понтрягина для этой задачи оптимального управления, получим, что существует абсолютно непрерьвная функция $\psi:\left[t_{0}, T\right] \rightarrow \mathbb{R}^{n *}$, для которой имеет место формула (3.4) и правая часть неравенства (3.6). Аналогично, фиксируя управление первого игрока $\widehat{u}(t)$ и рассматривая задачу максимизации функционала $\mathscr{J}$ по $v(t)$, получим существование функции $\widetilde{\psi}$, для которой выполнена формула (3.4) и левая часть неравенства (3.6), следовательно, функции $\psi$ и $\widetilde{\psi}$ совпадают и выполнены формулы (3.4)-(3.6).

Достаточность. Пусть функции $\widehat{x}, \widehat{u}, \widehat{v}, \psi$ удовлетворяют условиям (3.4)-(3.6). Обозначим $\bar{u}=\widehat{u}, \bar{v}=\widehat{v}, \bar{x}=\widehat{x}$ и определим $f_{x}, \zeta_{x}$ по формулам (2.3). Из (3.4) имеем

$$
\frac{d \psi(t)}{d t}=-\psi(t) f_{x}(t)-\zeta_{x}(t), \quad \psi(T)=\frac{d \gamma}{d x}(\widehat{x}(T))
$$

следовательно,

$$
\psi(t)=\frac{d \gamma}{d x}(\widehat{x}(T)) \Phi(t, T)+\int_{t}^{T} \zeta_{x}(\tau) \Phi(t, \tau) d \tau
$$

Откуда и из (2.6) получаем $\mathscr{D}(t)=\psi(t) f_{u}(t)$.

Из правой части неравенства (3.6) следует, что $0 \in \partial_{u} \mathscr{H}(t, \widehat{x}(t), \widehat{u}(t), \widehat{v}(t), \psi(t))$, поэтому $\mathscr{D}(t) \in-\partial_{u} \zeta(t, \widehat{x}(t), \widehat{v}(t), \widehat{u}(t))$, откуда в силу (2.2) и леммы 1 для любого $u \in \mathscr{U}$ получаем

$$
\int_{t_{0}}^{T}(\zeta(t, \widehat{x}(t), u(t), \widehat{v}(t))-\zeta(t, \widehat{x}(t), \widehat{u}(t), \widehat{v}(t))) d t \geqslant-\int_{t_{0}}^{T} \mathscr{D}(t)(u(t)-\widehat{u}(t)) d t+\frac{1}{2} \rho^{2}(u-\widehat{u}) .
$$

Откуда в силу леммы 2 и неравенства (1.12) имеем

$$
\begin{aligned}
& \mathscr{J}\left(t_{0}, x_{0}, \widehat{u}, \widehat{v}\right)-\mathscr{J}\left(t_{0}, x_{0}, u, \widehat{v}\right) \\
& \quad \leqslant \Delta \mathscr{J}\left(x_{0}, u\right)+\int_{t_{0}}^{T}(\zeta(t, \widehat{x}(t), u(t), \widehat{v}(t))-\zeta(t, \widehat{x}(t), \widehat{u}(t), \widehat{v}(t))) d t \leqslant 0 .
\end{aligned}
$$

Аналогично, для любого $v \in \mathscr{V} \mathscr{J}\left(t_{0}, x_{0}, \widehat{u}, \widehat{v}\right) \geqslant \mathscr{J}\left(t_{0}, x_{0}, \widehat{u}, v\right)$, т.е. управления $\widehat{u}, \widehat{v}$ являются оптимальными программными стратегиями. 
4. Пример. В данном пункте на простом примере сравнивается рассматриваемая формализация дифференциальных игр с сильно вьпукло-вогнутым интегрантом с другими классами дифференциальных игр.

Пусть динамика управляемого объекта определяется системой уравнений

$$
\begin{aligned}
& \dot{x}_{1}=x_{2}+u, \quad x_{1}(0)=x_{1}^{0}, \\
& \dot{x}_{2}=-x_{1}+v, \quad x_{2}(0)=x_{2}^{0}, \quad x_{1}, x_{2}, u, v \in \mathbb{R}, \quad t \in[0,2 \pi],
\end{aligned}
$$

функционал качества имеет вид

$$
\mathscr{J}=\frac{x_{1}^{2}(2 \pi)}{2}+2 \int_{0}^{2 \pi} u^{2}(t) d t-2 b \int_{0}^{2 \pi} v^{2}(t) d t
$$

на управление игрока $v$ наложено геометрическое ограничение $|v(t)| \leqslant c$, где $b, c>0-$ заданные константы.

Как известно, линейную систему с помощью линейного преобразования фазового вектора можно свести к системе, правая часть которой не зависит от фазового вектоpa. Обозначим $y(t)=x_{2}(t) \sin t-x_{1}(t) \cos t, y_{0}=-x_{1}^{0}$. Тогда

$$
\begin{gathered}
\dot{y}=v \sin t-u \cos t, \quad y(0)=y_{0} \\
\mathscr{J}=\frac{y^{2}(2 \pi)}{2}+2 \int_{0}^{2 \pi} u^{2}(t) d t-2 b \int_{0}^{2 \pi} v^{2}(t) d t .
\end{gathered}
$$

Проверим вьполнение предположений п. 1 .

В данном примере $\gamma(x)=\frac{1}{2} x^{2}$, поэтому $R^{+\gamma}=0, R^{-\gamma}=1, K_{u u}^{J}=0<1, K_{v v}^{J}=K_{v}^{f}$. Далее замечаем, что $e(t)=1, N_{v}^{f}(t)=\sin t, R_{v}^{\zeta}(t)=4 b$, следовательно, $K_{v v}^{J}=\pi /(4 b)$ и условие $K_{v v}^{J}<1$ выполняется при $b>\pi / 4$.

Для построения оптимальных стратегий воспользуемся принципом минимакса (теорема 3). Функция Понтрягина в нашем примере имеет вид

$$
\mathscr{H}(t, x, u, v, \psi)= \begin{cases}\psi v \sin t-\psi u \cos t+2 u^{2}-2 b v^{2}, & |v| \leqslant c \\ +\infty, & |v|>c .\end{cases}
$$

Так как $\partial \mathscr{H} / \partial x=0$, то

$$
\psi(t) \equiv \psi=\widehat{y}(2 \pi)
$$

и оптимальные программные стратегии имеют вид

$$
\begin{gathered}
\widehat{u}(t)=\frac{1}{4} \psi \cos t, \\
\widehat{v}(t)= \begin{cases}\frac{\psi \sin t}{4 b}, & |\psi \sin t| \leqslant 4 b c, \\
c \operatorname{sgn}(\psi \sin t), & |\psi \sin t|>4 b c .\end{cases}
\end{gathered}
$$

Подставляя оптимальные стратегии в уравнение динамики (4.1), находим оптимальную траекторию $\widehat{y}(t)$ :

$$
\widehat{y}(t)=y_{0}-\frac{\psi}{8}\left(t+\frac{\sin 2 t}{2}\right)+ \begin{cases}\frac{\psi}{8 b}\left(t-\frac{\sin 2 t}{2}\right), & |\psi| \leqslant 4 b c \\ \bar{y}(t) \operatorname{sgn} \psi, & |\psi|>4 b c\end{cases}
$$


где

$$
\bar{y}(t)= \begin{cases}\frac{|\psi|}{8 b}\left(t-\frac{\sin 2 t}{2}\right), & t \in\left[0, t_{\psi}\right], \\ \frac{|\psi|}{8 b} t_{\psi}+\frac{c}{2} \sqrt{1-\left(\frac{4 b c}{\psi}\right)^{2}}-c \cos t, & t \in\left[t_{\psi}, \pi-t_{\psi}\right], \\ \frac{|\psi| \psi \mid}{8 b} t_{\psi}+\frac{3}{2} c \sqrt{1-\left(\frac{4 b c}{\psi}\right)^{2}}+c \cos t, & t \in\left[\pi-t_{\psi}, \pi+t_{\psi}\right], \\ \frac{|\psi|}{8 b}\left(t-\frac{\sin 2 t}{2}-2 \pi+4 t_{\psi}\right)+2 c \sqrt{1-\left(\frac{4 b c}{\psi}\right)^{2}}, & t \in\left[\pi+t_{\psi}, 2 \pi-t_{\psi}\right], \\ t_{\psi}=\arcsin \frac{4 b c}{|\psi|} & t \in\left[2 \pi-t_{\psi}, 2 \pi\right],\end{cases}
$$

Откуда и из (4.3) получаем уравнение для определения $\psi$

$$
y_{0}= \begin{cases}\left(1+\frac{\pi}{4}-\frac{\pi}{4 b}\right) \psi, & |\psi| \leqslant 4 b c \\ \left(1+\frac{\pi}{4}\right) \psi-\frac{\psi}{2 b} \arcsin \frac{4 b c}{|\psi|}-2 c \sqrt{1-\left(\frac{4 b c}{\psi}\right)^{2}} \operatorname{sgn} \psi, & |\psi|>4 b c\end{cases}
$$

Из формул $(4.2),(4.4),(4.5)$ определим функцию цены игры $\Upsilon\left(0, y_{0}\right)=\mathscr{J}\left(0, y_{0}, \widehat{u}, \widehat{v}\right)(3.1)$ :

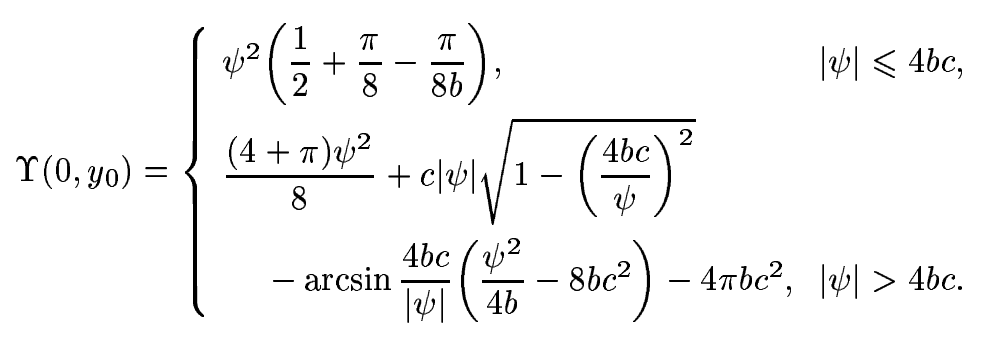

Как следует из теоремы 1, в случае $b>\pi / 4$ оптимальные программные стратегии игроков, оптимальная траектория и функция цены игры определяются формулами (4.4)-(4.6), (4.8), где вектор $\psi$ определяется по начальной позиции фазового вектора $y_{0}$ из уравнения (4.7).

В предельном случае $b=1, c=+\infty$ получаем линейно-квадратичную дифференциальную игру, решение которой может быть получено как по формулам (4.4)-(4.8), так и с помощью известного алгоритма, основанного на решении матричного дифференциального уравнения Риккати [3, с. 160]. Линейно-квадратичная формализация, как известно, не позволяет учитьвать геометрические ограничения на управления, так как при достаточно большом значении фазового вектора управления игроков будут сколь 

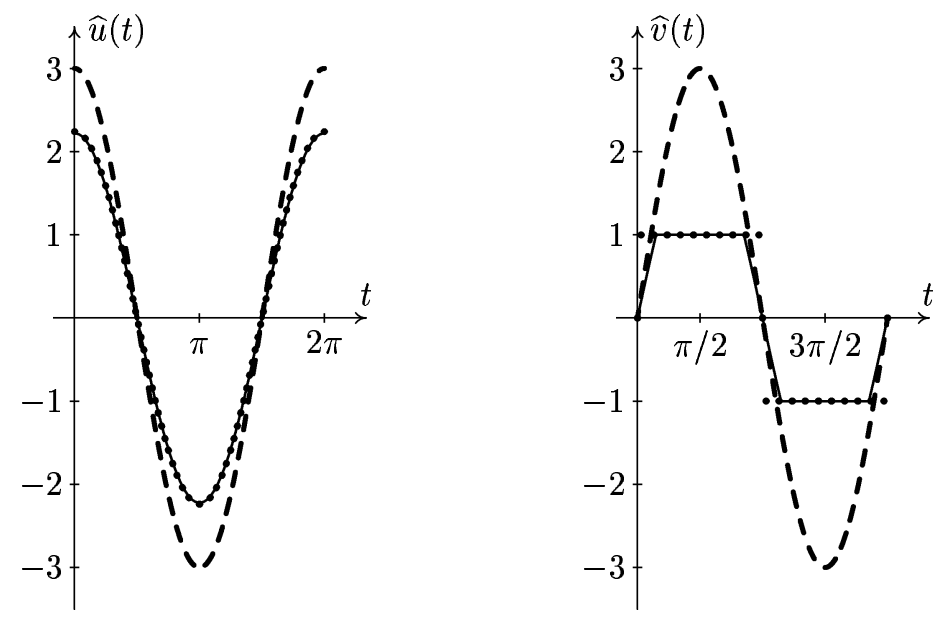

Р ис. 1. Оптимальные программные управления для игр с начальным условием $y_{0}=12$

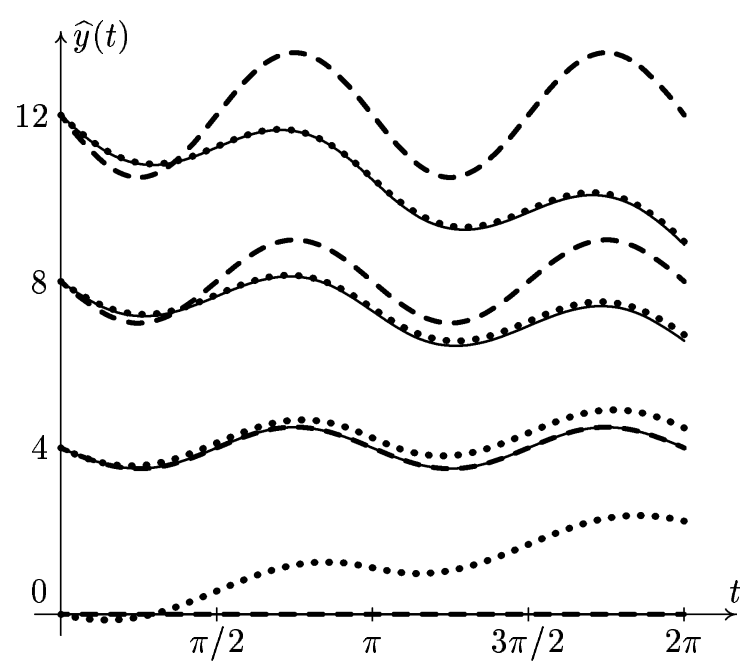

Рис. 2. Траектории, полученные из принщипа минимак$\mathrm{ca}$, для игр с начальными условиями $y_{0}=0,4,8,12$. Траектория для игры с параметрами $y_{0}=0, b=0, c=1$ не является оптимальной, остальные - оптимальные

угодно большими. На рис. 1-3 изображены оптимальные программные стратегии игроков, оптимальные траектории и функция цены игры. Графики, относящиеся к игре с параметрами $b=1, c=+\infty$, т.е. к линейно-квадратичной игре, показаны пунктиром.

В случае $b=0, c=1$ на управление игрока $v$ наложено лишь геометрическое ограничение. В этом случае предположение п. 1 о сильной выпукло-вогнутости интегранта не вьполнено. Если все же седловая точка в классе программных стратегий существует, то имеет место принцип минимакса (теорема 3). Тогда оптимальные стратегии, 


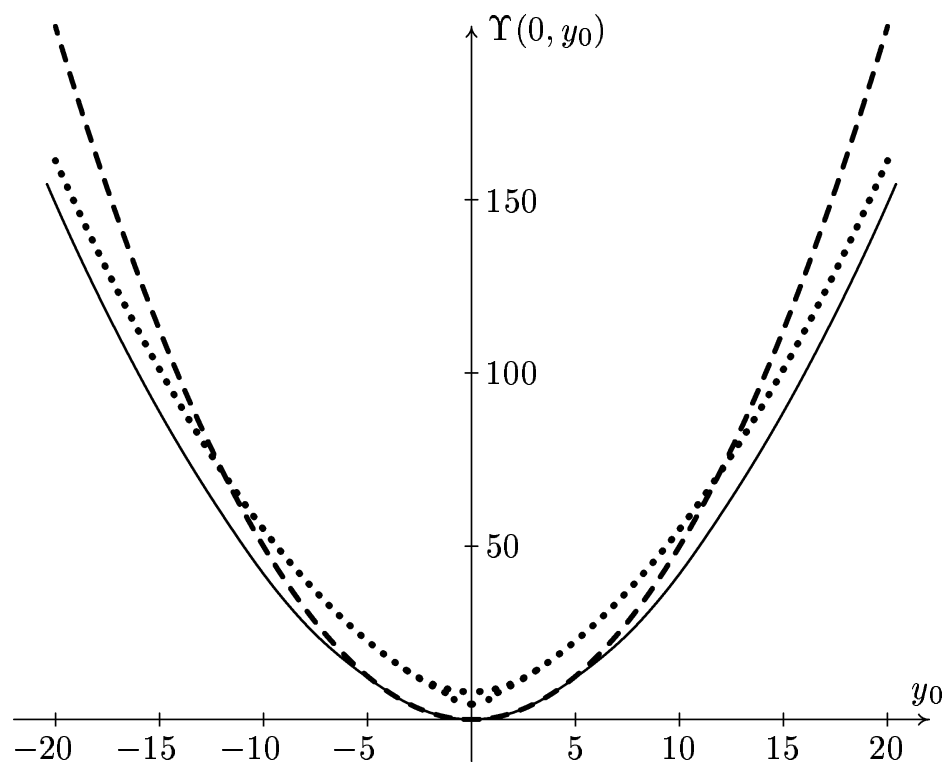

Рис. 3. Функции цен дифференциальных игр

оптимальная траектория и функция цены игры определяются формулами

$$
\begin{gathered}
\widehat{u}(t)=\frac{1}{4} \psi \cos t, \quad \widehat{v}(t)=\operatorname{sgn}(\psi \sin t), \\
\widehat{y}(t)=y_{0}-\frac{\psi}{8}\left(t+\frac{\sin 2 t}{2}\right)+\operatorname{sgn} \psi \cdot \begin{cases}1-\cos t, & t \in[0, \pi], \\
3+\cos t, & t \in[\pi, 2 \pi],\end{cases}
\end{gathered}
$$

следовательно,

$$
\begin{gathered}
\psi=\frac{\left|y_{0}\right|+4}{1+\pi / 4} \operatorname{sgn} y_{0}, \quad \widehat{u}(t)=\frac{\left|y_{0}\right|+4}{4+\pi} \cos t \operatorname{sgn} y_{0}, \quad \widehat{v}(t)=\operatorname{sgn}\left(y_{0} \sin t\right), \\
\widehat{y}(t)=y_{0}-\frac{\left|y_{0}\right|+4}{8+2 \pi}\left(t+\frac{\sin 2 t}{2}\right) \operatorname{sgn} y_{0}+\operatorname{sgn} y_{0} \cdot\left\{\begin{array}{l}
1-\cos t, \quad t \in[0, \pi], \\
3+\cos t, \quad t \in[\pi, 2 \pi],
\end{array}\right. \\
\Upsilon\left(0, y_{0}\right)=\frac{2\left(\left|y_{0}\right|+4\right)^{2}}{4+\pi} .
\end{gathered}
$$

Седловая точка в классе программных стратегий существует тогда и только тогда, когда

$$
\min _{u \in \mathscr{U}} \max _{v \in \mathscr{Y}} \mathscr{J}\left(0, y_{0}, u, v\right)=\max _{v \in \mathcal{Y}} \min _{u \in \mathscr{U}} \mathscr{J}\left(0, y_{0}, u, v\right) .
$$

Найдем $\max _{v \in \mathcal{V}} \min _{u \in \mathscr{U}} \mathscr{J}\left(0, y_{0}, u, v\right)$. При фиксированном программном управлении $v \in \mathscr{V}$ минимум $\mathscr{J}\left(0, y_{0}, u, v\right)$ по $u$ в силу принципа максимума Понтрягина достигается на функции $u(t)=\frac{1}{4} \psi \cos t$, где

$$
\psi=y(2 \pi)=y_{0}-\frac{\pi}{4} \psi+\int_{0}^{2 \pi} v(t) \sin t d t,
$$


поэтому

$$
\begin{gathered}
\min _{u \in \mathscr{U}} \mathscr{J}\left(0, y_{0}, u, v\right)=\frac{4+\pi}{8} \psi^{2}=\frac{2}{4+\pi}\left(y_{0}+\int_{0}^{2 \pi} v(t) \sin t d t\right)^{2}, \\
\max _{v \in \mathscr{V}} \min _{u \in \mathscr{U}} \mathscr{J}\left(0, y_{0}, u, v\right)=\max _{|V| \leqslant 4} \frac{2\left(y_{0}+V\right)^{2}}{4+\pi}=\frac{2\left(\left|y_{0}\right|+4\right)^{2}}{4+\pi} .
\end{gathered}
$$

Найдем $\min _{u \in \mathscr{U}} \max _{v \in \mathcal{V}} \mathscr{J}\left(0, y_{0}, u, v\right)$ :

$$
\begin{aligned}
\mathscr{J}_{\max }\left(y_{0}, u\right) & \equiv \max _{v \in \mathcal{V}} \mathscr{J}\left(0, y_{0}, u, v\right) \\
& =\frac{1}{2} \max _{|V| \leqslant 4}\left(y_{0}-\int_{0}^{2 \pi} u(t) \cos t d t+V\right)^{2}+2 \int_{0}^{2 \pi} u^{2}(t) d t \\
& =\frac{1}{2}\left(\left|y_{0}-\int_{0}^{2 \pi} u(t) \cos t d t\right|+4\right)^{2}+2 \int_{0}^{2 \pi} u^{2}(t) d t .
\end{aligned}
$$

Для определения $u_{\min \max }$ - функции, на которой достигается минимум по $u \in \mathscr{U}$ функционала $\mathscr{J}_{\max }\left(y_{0}, u\right)$ - рассмотрим два случая:
1) $\int_{0}^{2 \pi} u_{\min \max }(t) \cos t d t=y_{0}$;
2) $\int_{0}^{2 \pi} u_{\min \max }(t) \cos t d t \neq y_{0}$.

В случае 1) согласно принципу максимума Понтрягина

$$
u_{\min \max }(t)=\frac{y_{0} \cos t}{\pi}, \quad \min _{u \in \mathscr{U}} \mathscr{J}_{\max }\left(y_{0}, u\right)=8+\frac{2 y_{0}^{2}}{\pi} \equiv \widehat{\mathscr{J}}_{1}\left(y_{0}\right) .
$$

В случае 2)

$$
\begin{gathered}
u_{\min \max }(t)=\frac{\left|y_{0}\right|+4}{4+\pi} \cos t \operatorname{sgn} y_{0}, \\
\min _{u \in \mathscr{U}} \mathscr{J}_{\max }\left(y_{0}, u\right)=\frac{1}{2}\left(\frac{4|| y_{0}|-\pi|}{4+\pi}+4\right)^{2}+\frac{2 \pi\left(\left|y_{0}\right|+4\right)^{2}}{(4+\pi)^{2}} \equiv \widehat{\mathscr{J}}_{2}\left(y_{0}\right) .
\end{gathered}
$$

Так как при $\left|y_{0}\right|<\pi$ вьполнено неравенство $\widehat{\mathscr{J}}_{1}\left(y_{0}\right)<\widehat{\mathscr{J}}_{2}\left(y_{0}\right)$, а при $\left|y_{0}\right|>\pi-$ неравенство $\widehat{\mathscr{J}}_{1}\left(y_{0}\right)>\widehat{\mathscr{J}}_{2}\left(y_{0}\right)$, то при $\left|y_{0}\right|<\pi$ реализуется случай 1$)$, а при $\left|y_{0}\right|>\pi-$ случай 2). Следовательно,

$$
\min _{u \in \mathscr{U}} \max _{v \in \mathcal{V}} \mathscr{J}\left(0, y_{0}, u, v\right)= \begin{cases}8+\frac{2}{\pi} y_{0}^{2}, & \left|y_{0}\right|<\pi \\ \frac{2\left(\left|y_{0}\right|+4\right)^{2}}{4+\pi}, & \left|y_{0}\right| \geqslant \pi .\end{cases}
$$

Из формул (4.10), (4.11) следует, что седловая точка в классепрограммных стратегий для игры с параметрами $b=0, c=1$ существует только при $\left|y_{0}\right| \geqslant \pi$.

Графики, относящиеся к дифференциальной игре с параметрами $b=0, c=1$, т.е. с чисто геометрическими ограничениями на управление игрока $v$, показаны на рис. $1-3$ линией из точек. 
На рис. 1 изображены оптимальные программные управления игроков для игры с начальньм условием $y_{0}=12$. Оптимальная программная стратегия игрока $v$ имеет разрьв в точке $t=\pi$. На рис. 2 изображены траектории фазового вектора, полученные из принципа минимакса по формуле (4.9) для игр с начальными условиями $y_{0}=0,4,8,12$. Траектория, соответствующая $y_{0}=0$, не является оптимальной (при $\left|y_{0}\right|<\pi$ оптимальных траекторий не существует), а остальные траектории - оптимальные. На рис. 3 изображены графики функций

$$
\max _{v \in \mathcal{V}} \min _{u \in \mathscr{U}} \mathscr{J}\left(0, y_{0}, u, v\right) \quad \text { и } \quad \min _{u \in \mathscr{U}} \max _{v \in \mathcal{V}} \mathscr{J}\left(0, y_{0}, u, v\right) .
$$

При $\left|y_{0}\right| \geqslant \pi$ значения этих функций совпадают со значением цены игры. При $\left|y_{0}\right|<\pi$ значение цены игры [3, с. 78] лежит между значениями (4.12).

Сплошной линией на рис. 1-3 изображены оптимальные программные стратегии игроков, оптимальные фазовые траектории и функция цены для игры с параметрами $b=1$, $c=1$, полученные по формулам (4.4)-(4.6), (4.8). Для такой формализации дифференциальной игры, с одной стороны, явно учтены геометрические ограничения на управление игрока $v$, а с другой стороны, вьполнены условия сильной выпукло-вогнутости интегранта, что позволяет применить принцип минимакса Понтрягина.

Как видно из рассмотренного примера, линейная дифференциальная игра с сильно выпукло-вогнутьм интегрантом при малых значениях фазового вектора близка к линейно-квадратичной игре, а при больших значениях фазового вектора - к игре с чисто геометрическими ограничениями на управление.

\section{СПИСОК ЦИТИРОВАННОЙ ЛИТЕРАТУРЫ}

[1] Айзекс Р. Дифференциальные игры. М.: Мир, 1967.

[2] Понтрягин Л.С. Линейные дифференциальные игры преследования // Матем. сб. 1980. T. 112. № 3. C. 307-330.

[3] Красовский Н. Н. Управление динамической системой. М.: Наука, 1985.

[4] Rockafellar R. T. Integral functionals, normal integrands and measurable selections // Lecture Notes in Math. 1976. V. 543. P. 157-207.

[5] Экланд И., Темам Р. Выпуклый анализ и вариационные проблемы. М.: Мир, 1979.

[6] Колмогоров А. Н., Фомин С. В. Элементы теории функций и функционального анализа. М.: Наука, 1989.

[7] Нейман Дж. фон. К теории стратегических игр // Матричные игры. М.: Физматгиз, 1961. C. $173-204$. 